Rev. Elev. Méd. vét. Pays trop., 1971, 24 (4) : $667-87$

\title{
Le Kouri : race bovine du lac Tchad I. Introduction générale à son étude zootechnique et biochimique : origines et écologie de la race
}

\author{
par R. QUEVAL (*), J. P. PETIT (**), G. TACHER (*), \\ A. PROVOST $(*)$ et J. PAGOT $(* *)$
}

\begin{abstract}
RESUME
Les auteurs présentent de façon détaillée la race des taurins Kouris, bovins du lac Tchad. Cette première étude est l'introduction pour l'ensemble des travaux qui visent à éclairer les divers aspects zootechniques et biochimiques de cette race, connue depuis longtemps déjà et qui est étroitement liée au milieu aquatique du lac Tchad.

Le but poursuivi est de ne pas laisser disparaître un maillon, sans doute essentiel, dans la phylogénie des races bovines et de tirer profit rapidement des propriétés les plus intéressantes de la race: production laitière, production de viande de qualité et propriétés des métis de $1^{\text {re }}$ ou de 2 e génération.

Après l'étude écologique de l'aire géographique, le type caractéristique est décrit ainsi que ses propriétés économiques.

Le mode d'élevage et la pathologie sont largement détaillés.

De nombreuses raisons plaident actuellement pour le maintien et la sélection des bovins Kouris purs.
\end{abstract}

Parmi les types et races des bovins d'Afrique, un taurin se distingue en raison de ses particularités ethniques et de ses aptitudes, il repré. sente un des types de bovidés les plus intéressants du continent africain: le bœuf du lac Tchad.

Ce taurin, appelé généralement Kouri, est connu également sous les dénominations de bœuf Boudouma, Koubouri, Dongolé, Borrie ou Baré.

Ce bœuf sans bosse, à cornage renflé très caractéristique, a pour berceau les îles et les berges marécageuses du lac Tchad.

Aujourd'hui, cette race autochtone est menacée de disparition ou de «dilution » par l'ap-

(*) I.E.M.V.T., Laboratoire de Recherches vétérinaires de Farcha, B.P. no 433, Fort-Lamy, Tchad.

(**) I.E.M.V.T., 10, rue Pierre Curie - 94 MaisonsAlfort. port de sang étranger tel que celui du Zébu arabe ou Bororo.

Cette étude a pour but de recueillir les données, renseignements et observations de tous ordres, zootechniques et biochimiques en particulier, concernant la race Kouri pure.

Limportance du sujet oblige à le fractionner en études précises et complémentaires.

L'objectif de la présente note est de rassembler et de trier les éléments, en majeure partie zootechniques, antérieurement publiés, et de présenter une mise au point générale relative à la race (origine; milieu: géographique, humain et naturel (climatologie, géologie, végétation); caractères ethniques; aptitudes et pathologie.

Cette mise au point générale sera complétée et mise à jour ultérieurement par des publica- 
tions analysant les résultats de recherches biologiques, génétiques et biochimiques. Celles-ci ont pour objet : les groupes sanguins (détermination de la nature et de la fréquence des facteurs érythrocytaires, ainsi que des allèles, des locus complexes, la détermination de la nature des hémoglobines en recherchant ses incidences génétiques, les normes biologiques, ionogramme, protéinogramme, lipidogramme, glucidogramme, etc.); enfin une étude biométrique.

Le bilan des investigations sur le Kouri peut permettre la mise en évidence de propriétés biologiques spécifiques à la race, éventuellement les potentialités intéressantes de ces taurins et ainsi d'orienter leur devenir.

En effet, cette race, outre les problèmes biologiques d'adaptation au milieu, offre de nombreux centres d'intérêt parmi lesquels nous ne retiendrons que deux points : l'un économique, l'autre scientifique.

Du point de vue économique, l'hypothèque posée sur l'élevage par la pathologie tend de plus en plus à être levée et, dans ces conditions, l'accroissement de la production animale par l'amélioration de l'alimentation et par l'action zootechnique devient possible. Tant en la sélectionnant qu'en la métissant, la race Kouri de par ses potentialités pourrait présenter un intérêt certain. Le métis zébu-bœuf du lac, entretenu par les ethnies Kanembou et Haddad. présente des aptitudes laitière et bouchère supérieures à celles du zébu arabe.

D'un point de vue scientifíque, l'étude des groupes sanguins et l'analyse de certains locus peuvent contribuer à éclairer la phylogénie des races bovines africaines.

\section{PROVENANCE}

Les Kouris ou boufs du lac Tchad se caractérisent par d'énormes cornes bulbeuses, une robe blanche et l'absence de bosse. Ils ont été considérés comme un groupe distinct dans la classification de R. JOSHI, A. McLAUGHLIN et W. PHILLIPS (1957) (7) où ils constituent le groupe IV.

\section{Légende}

Quatre légendes relatives à l'origine du bœuf du lac Tchad ont été rapportées par KASSOUM KONE (1948) (8).

De nos jours, les vieux éleveurs Boudoumas racontent qu'un arabe venant du Sud Yémen se rendit au lac Tchad, gagna les îles de Tagal au nord-ouest de Baguirmi et remarqua des traces et empreintes d'animaux sur ces îles.

$\mathrm{Au}$ soleil couchant, dans le crépuscule, le voyageur vit sortir des roseaux du lac de grands bœufs à grosses cornes. L'homme alluma un feu, les animaux se rassemblèrent autour, et ne quittèrent plus leur premier pasteur.

\section{Origine}

Les diverses hypothèses sur l'origine obscure de cette race ont été analysées par MALBRANT, RECEVEUR et SABIN en 1947 (11) et sont résumées ci-dessous.

BARON considère le bœuf du lac Tchad comme une race bien définie, Bos taurus belensis, possédant de grandes ressemblances avec la grande race grise des steppes d'Asie, qui elle-même serait originaire d'Afrique.

PECAUD estime que cet animal est un descendant des taurins Peuhls à robe blanche qui se seraient modifiés par l'adaptation à un nouveau genre de vie.

STEWART (1938) (17) suppose que ce taurin est identique à l'Hamitique à longues cornes des dessins égyptiens. La tradition rapporte que ces animaux furent importés environ 2.000 ans avant Jésus-Christ par les envahisseurs venus du Nil qui, défaits, se réfugièrent dans les îles du lac Tchad, ce qui expliquerait la pureté de Ia race.

EPSTEIN (1936) et CURSON (1936) (2), sur la foi de seuls documents photographiques, considèrent ce bœuf comme un animal à bosse et le rangent dans la catégorie des pseudo-zébus ou Sanga. Selon CURSON, vers 3 ou 4.000 ans avant Jésus-Christ, les égyptiens domestiquèrent le bœuf sauvage à grandes cornes de la Vallée du Nil descendant du Bos primigenius Hahni.

A la fin du néolithique, un taurin originaire d'Asie, le Bos brachyceros, fut introduit sur le continent africain; simultanément, on observa le refoulement et l'émigration vers l'ouest africain de Bos primigenius. Vers 1.000 ans avant Jésus-Christ, un zébu asiatique à cornes 


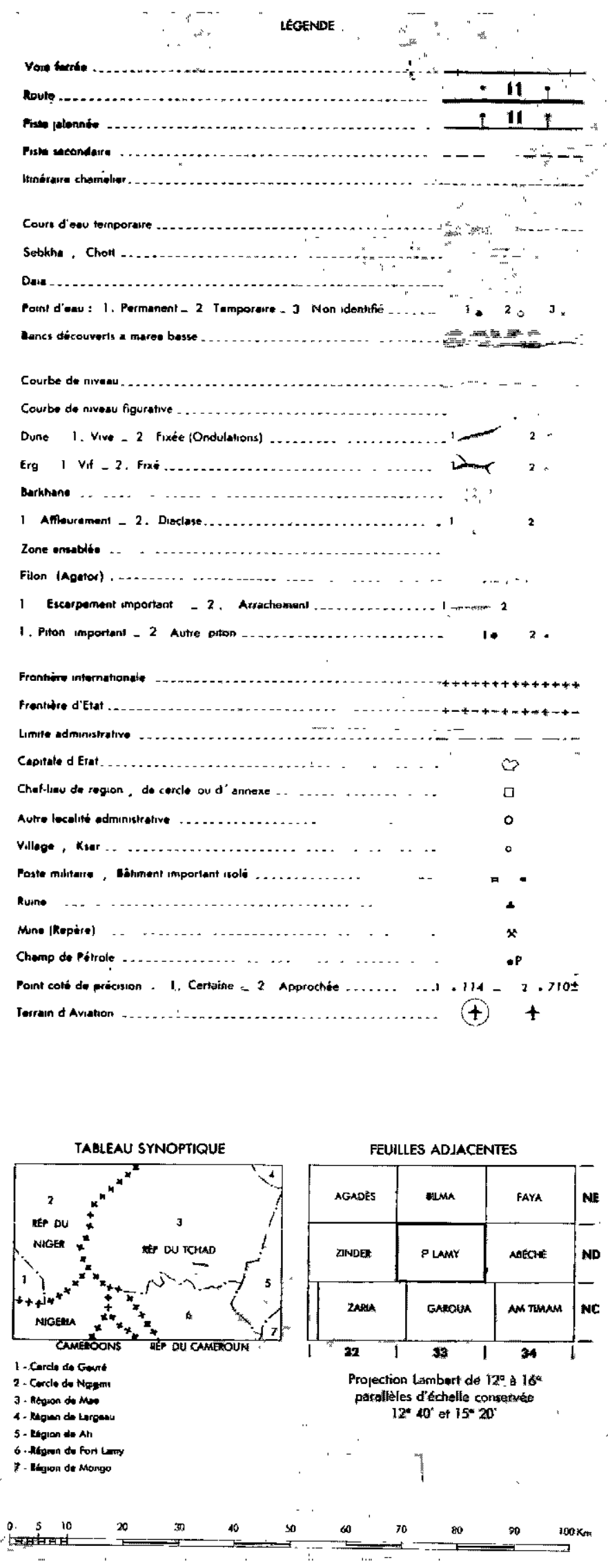

Autorisation I.G.N. n $\mathrm{n}^{\circ} 90103$

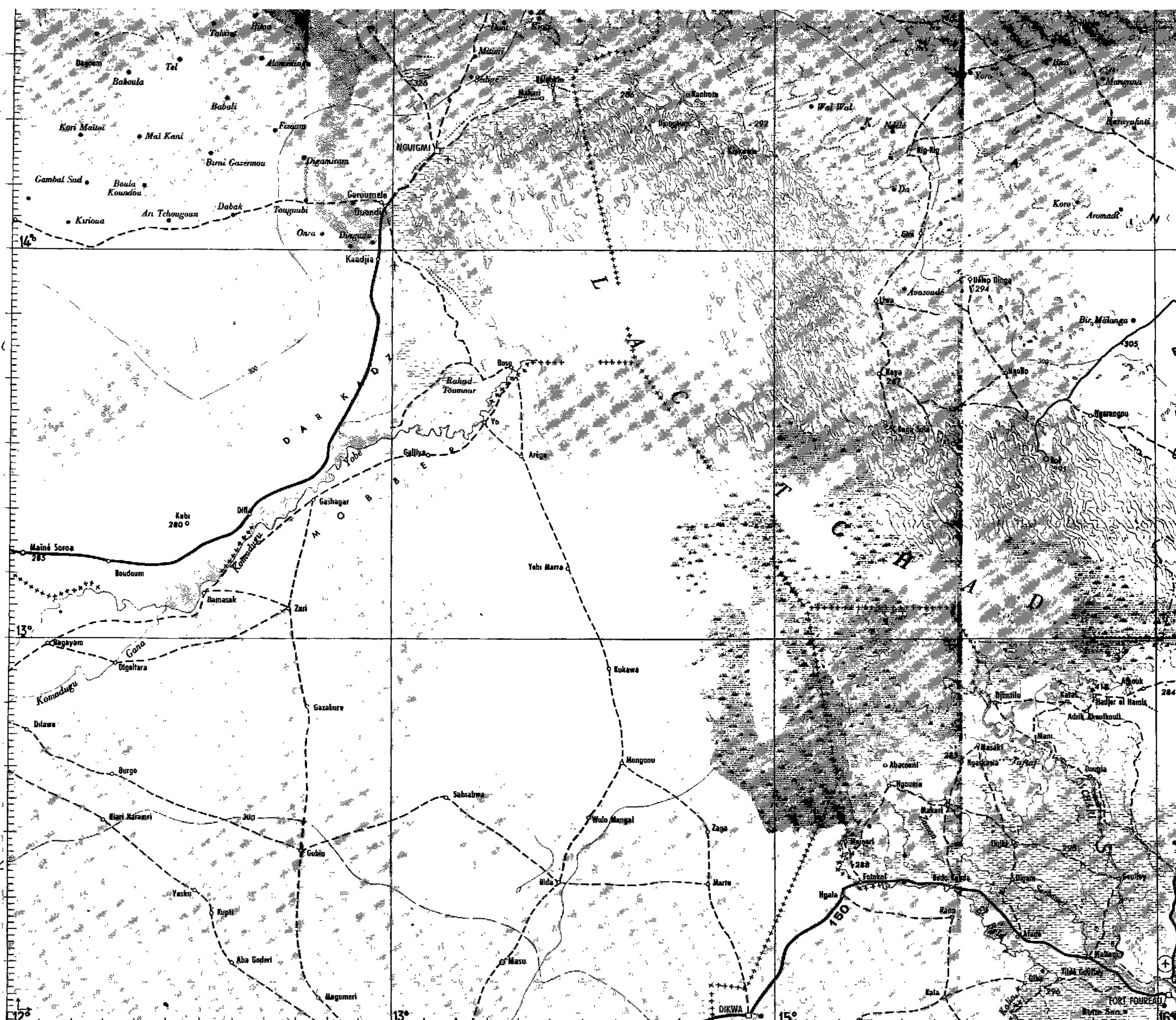

El 


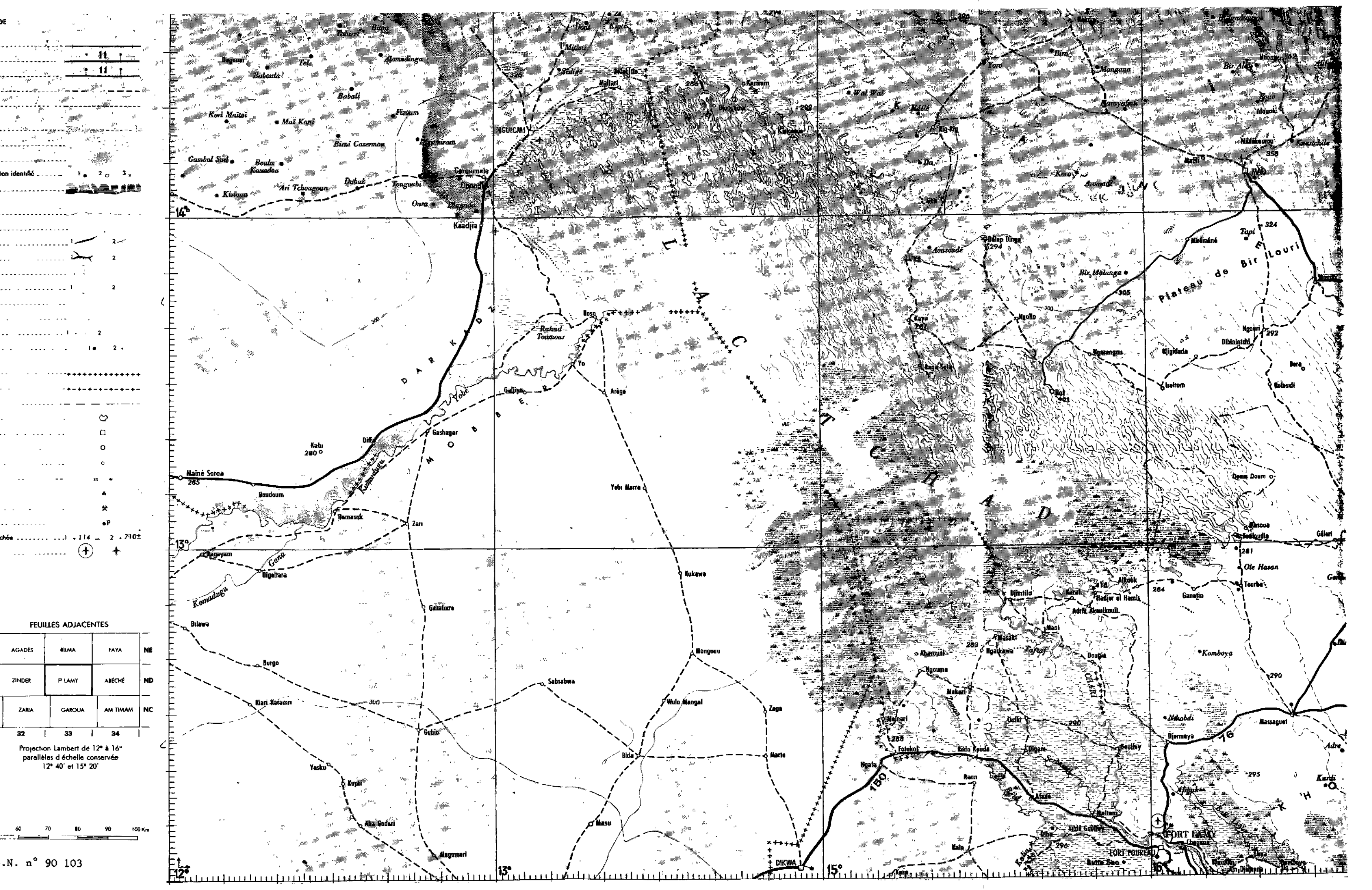


latérales et à bosse musculaire située en position cervico-dorsale fut introduit en Afrique. Aujourd'hui cette souche pure constitue la race Afrikander d'Afrique du Sud.

Le croisement du zébu à cornes latérales avec le Bos primigenius dans le haut bassin du Nil au début de l'ère chrétienne aboutit à la constitution de la race Sanga dont le bouf du lac Tchad ne serait qu'un descendant.

\section{MILIEU}

\section{Milieu géographique}

La race taurine Kouri est une race stabilisée. Son berceau se situe au lac Tchad, reste de la mer paléo-tchadienne, comprise entre $12^{\circ} 20^{\prime}$ et $14^{\circ} 20^{\prime}$ de latitude nord d'une part, et entre $13^{\circ}$ et $15^{\circ} 30^{\prime}$ de longitude est, d'autre part.

L'élevage de ces taurins, bien adaptés au milieu, est intimement lié aux caractéristiques hydrologiques du lac.

Le lac Tchad est par ordre d'importance le $11^{\mathrm{e}} \mathrm{du}$ monde et se partage entre les territoires de quatre états : le Tchad à l'Est, la République du Niger au Nord-Ouest, la Fédération du Nigéria à l'Ouest et le Cameroun au Sud.

La morphologie du lac comprend 3 parties '

- deux zones d'eaux libres, lune au NordOuest, l'autre au Sud, à l'embouchure du Chari; elles sont séparées par la "grande barrière " d'orientation Nord-Est - SudOuest. Cette dernière étant un barrage végétal où sur les hauts fonds se sont développés les papyrus et les roseaux;

- l'archipel tout le long de la rive Nord-Ouest, constitué par une multitude d'îles allongées dans le sens Nord-Ouest - Sud-Est. Ce sont les vestiges d'un réseau de dunes partiellement submergées. Cet archipel est séparé des eaux libres du Sud par les îlots bancs qui sont les sommets d'anciennes dunes, immergés sous une faible profondeur d'eau, et recouverts d'une végétation aquatique. Les archipels du Nord-Est sont des dunes de sable qui pénètrent dans le lac, aussi, sur 10 ou $15 \mathrm{~km}$, le littoral est découpé par une multitude de canaux lagunaires. Par contre, la côte Nord-Ouest (Niger et Nigéria) est franche;

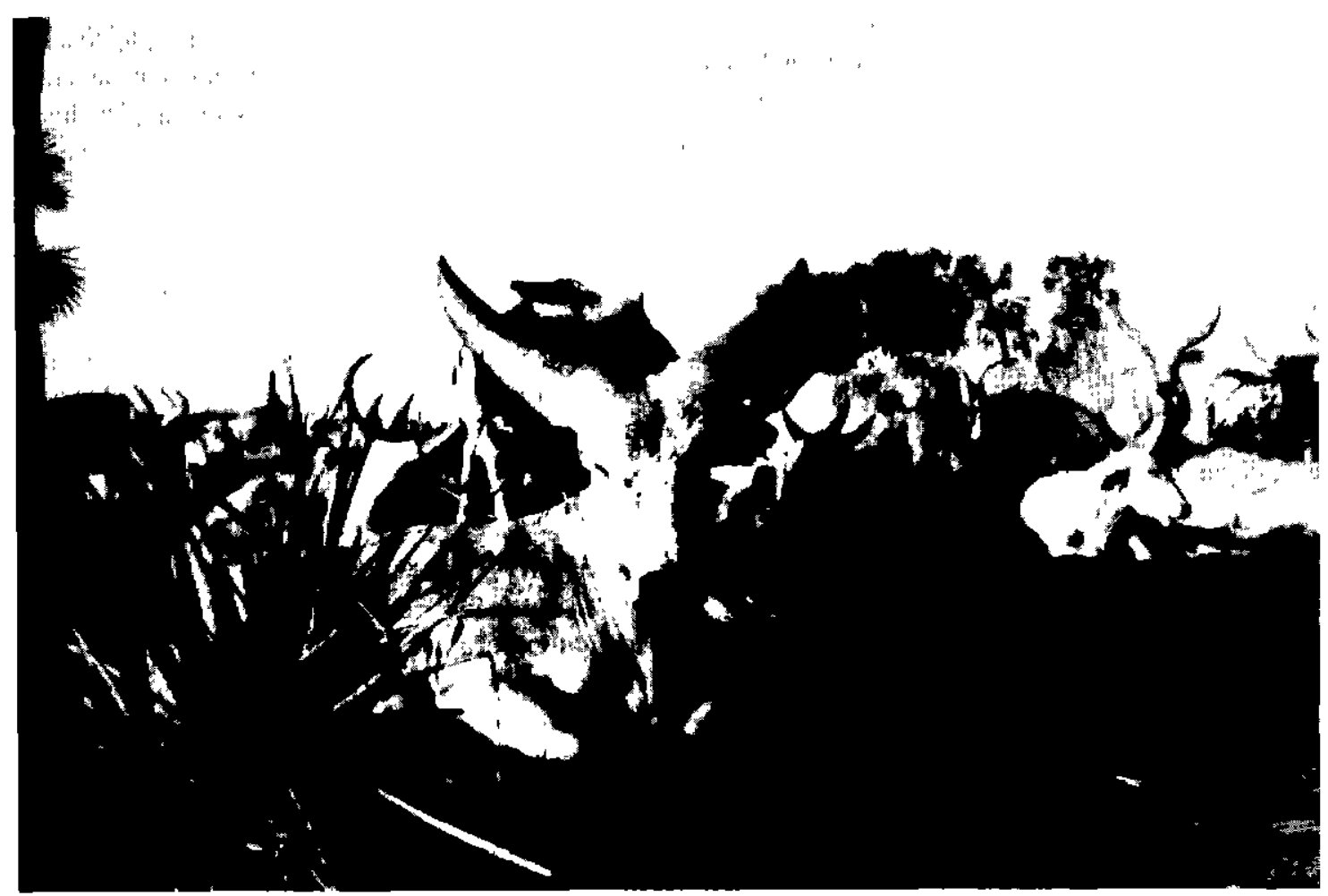

Photo no 1 - Kouris dans leur enviconnement spécifique. 
- deux zones de marécages ou herbiers situées respectivement au Sud-Ouest et à l'Est des eaux libres du Sud.

Ce lac, véritable mer intérieure, d'une superficie oscillant de 10 à $26.000 \mathrm{~km}^{2}$ à une altitude moyenne de 280 mètres, est alimenté, pour la plus grande part, par le Chari $(1.200 \mathrm{~km})$ et le Logone $(970 \mathrm{~km})$, qui recueillent les pluies des montagnes camerounaises et centrafricaines.

Le lac est relativement peu profond $(0$ à 10 mètres) et son niveau varie de 2 à 3 mètres entre la saison sèche et la saison humide. Il est arrivé qu'au cours d'une période de 30 à 50 ans il se soit presque complètement asséché du fait de la réduction des apports d'eau provenant des variations de la pluviométrie.

Les fortes crues sont désastreuses pour l'élevage. Les inondations réduisent les surfaces pâturables, d'où une sous-alimentation et une plus grande sensibilité aux épizooties.

\section{Milieu humain}

La préfecture tchadienne du lac, située sur la bordure Est du lac Tchad, a une économie basée sur l'agriculture et l'élevage.

Les cultivateurs pratiquent sur les îles, et en particulier dans les polders, diverses cultures vivrières : variétés de mil (rouge, blanc, petit), maïs, blé, haricots, melons, arachides, cucurbitacées, piments etc... Le Gouvernement porte actuellement ses efforts sur la modernisation du secteur agricole dans cette région (irrigation, introduction de nouvelles cultures comme la pomme de terre, la canne à sucre, le coton...).

Les éleveurs se livrent à la pratique de l'agriculture.

Lc cheptcl bovin de la préfecture du lac est estimé à 270.000 têtes.

Le mode d'élevage est essentiellement séden. taire sur le continent et semi transhumant en saison sèche pour les animaux vivant sur les îles. Les insulaires et les riverains du lac Tchad sont :

- des descendants présumés des Sao : les Boudoumas et les Kouris. Ils se désignent eux-mêmes par le nom de Yédina;

- lcs Kanembous, race originaire du Yémen, d'où partiront vers le continent africain des guerriers. Ces Yéménites fondèrent à l'Ouest du lac le sultanat du Bornou. Du mélange de ces éléments avec les Sao légendaires naît le peuple Kanembou.

Les cantons lacustres sont peuplés en majorité de Boudoumas, et les cantons mi-lacustres mi-terriens peuplés de Kanembous et de Kouris.

Les Boudoumas seuls peuplent les îles basses de l'Ouest couvertes de pâturages. Ces éleveurs se déplacent par petits groupes, allant d'île en île, avec leurs troupeaux, à la recherche de nouveaux pâturages. Hommes et animaux franchissent à la nage les canaux lacustres. Femmes, hommes et enfants, vêtements roulés sur la tête, ont le corps immergé, allongé à califourchon sur un tronc d'arbre de densité deux fois moindre que celle du liège : le bois d'ambadj (Herminiera elaphroxylon). Ils vont et viennent parmi les animaux, les surveillent, les aident, les encouragent de la voix, les incitent à suivre le bœuf passeur.

Les jeunes et les petits ruminants sont transportés sur de vastes embarcations plates faites de papyrus, habilement bottelées et bien profilées : les Kadei.

Les éleveurs Boudoumas ne se réfugient dans les îles hautes ou sur le littoral que lorsque les eaux de crues ont partiellement inondé les bas pâturages. Dans le Sud, les troupeaux de l'ethnie Boudouma étant moins prospères, les habitants ont des bases plus sédentaires sur les îles où ils cultivent le mil en saison des pluies.

Les îles hautes en bordures du littoral sont peuplées à la fois par les Boudoumas et les Kanembous et au Sud par les Kouris. Ces derniers possèdent sur le sommet de leurs îles d'authentiques villages et pratiquent d'importantes cultures de mil, de blé et de maïs.

\section{Milieu naturel}

\section{A. Données climatologiques}

Ces données, relatives au régime des vents, de la température, de l'humidité et de la pluviométrie ont été extraites de la monographie hydrologique du lac Tchad, publiée par l'Office de la Recherche Scientifique et Technique Outre-Mer (1969) (18).

Les régions riveraines du lac reçoivent, en année moyenne, des précipitations comprises entre 250 et $550 \mathrm{~mm}$; leur climat est donc de 
type sahélien et confine vers le Nord au type subdésertique.

Les caractéristiques saisonnières de ces climats sont liées aux déplacements du front intertropical (FIT) qui sépare Ia masse d'air tropicale continentale (harmattan) de l'équatoriale maritime (mousson).

De novembre à mars, le FI'l' est situé au sud du lac : l'harmattan règne et la saison sèche est absolue. En avril, le FIT remonte lentement vers le Nord et de petites précipitations apparaissent en mai et juin. Le FIT atteint sa position la plus septentrionale vers le $20^{\prime \prime}$ parallèle en juillet et août. Les trois quarts des précipitations annuelles tombent pendant ces 2 mois. Puis, le FIT rétrograde vers le Sud, la saison sèche s'installe alors à la fin de septembre ou au début d'octobre.

Les caractéristiques climatiques suivantes ont été observées à la station météorologique de Bol ( $13^{\circ} 28^{\prime}$ de latitude nord et $14^{\circ} 43^{\prime}$ de longitude est, altitude 291 mètres).

a) Vents

Le régime des vents est lié d'une part aux déplacements du FIT et d'autre part aux phénomènes thermiques créés localement par la présence même du lac.

En saison sèche, entre octobre-novembre et avril, les vents soufflent du Nord - Nord-Est et de l'Est, déterminant l'harmattan, vent sec venant des régions désertiques qui transporte de fines particules donnant naissance à des brumes sèches.

En saison des pluies, de mai à septembre, la mousson vient du Sud-Ouest et de l'Ouest.

Les vents locaux secondaires sont :

- dans la journée, une brise venant du lac lorsque la température des régions riveraines est plus forte que sur le lac;

- la nuit, une brise venant des terres lorsque la température superficielle du lac est sensiblement plus élevée que celle des régions avoisinantes.

Les vents sont toujours et nettement plus forts le jour que la nuit.

b) Température

TABLEAU $\mathbf{N}^{*} \mathrm{I}$

Températuresmoyennes observées dans la région du lac Tchad.

\begin{tabular}{|c|c|c|c|c|c|c|c|c|c|c|c|c|}
\hline Mois & J & F & M & A & M & J & J & A & S & 0 & N & D \\
\hline Tx & 31,4 & 32,6 & 36,6 & 38,5 & 37,6 & 35,3 & 33,0 & 31,0 & 33,3 & 36,5 & 35,0 & 32,3 \\
$\mathrm{Tn}$ & 14,0 & 15,1 & 19,4 & 24,2 & 25,9 & 26,6 & 25,3 & 24,2 & 24,7 & 22,6 & 18,4 & 14,6 \\
$\mathrm{Tx}+\mathrm{Tn}$ & 22,7 & 23,0 & 28,0 & 31,3 & 31,8 & 31,0 & 29,2 & 27,6 & 29,0 & 29,6 & 26,7 & 23,5 \\
$\mathrm{Tx}-\mathrm{Tn}$ & 17,4 & 17,5 & 17,2 & 14,3 & 11,7 & 8,7 & 7,7 & 6,8 & 8,6 & 13,9 & 16,6 & 17,7 \\
\hline
\end{tabular}

Les moyennes mensuelles colligées dans le tableau $n^{\circ}$ I ont été calculées après une période d'observation de 13 ans (1954-1966) et sont relatives à :

- la moyenne mensuelle des températures maximales journalières $(\mathrm{Tx})$;

- la moyenne mensuelle des températures minimales journalières (Tn);

- la température moyenne mensuelle $\frac{\mathrm{Tx}+\mathrm{Tn}}{2}$;

- l'écart journalier moyen mensuel (Tx - Tn).
Les températures moyennes mensuelles présentent un minimum allant de $14^{\circ}$ en janvier à $26,6^{\circ}$ en juin et un maximum allant de $31^{\circ}$ en août à $38,5^{\circ}$ en avril. La température moyenne annuelle est de $27,8^{\circ}$.

c) Humidité

L'humidité relative moyenne ainsi que la tension de vapeur d'eau ont été calculées sur une période de 10 ans (1957-1966). Les résultats sont présentés dans le tableau $\mathrm{n}^{\circ}$ II.

Le maximum d'humidité relative se situe en août et le minimum en février-mars. 
TABLEAU $\mathrm{N}^{\circ} \mathrm{IT}$

Humidité relative et tension de vapeur d'eau moyennes dans la rëgion du lac Tchad

\begin{tabular}{|c|c|c|c|c|c|c|c|c|c|c|c|c|}
\hline Mois & J & F & M & A & M & J & J & A & S & 0 & N & D \\
\hline $\begin{array}{l}\text { Humidite } \\
\text { relative }\end{array}$ & 34 & 29 & 24 & 41 & 52 & 61 & 69 & 76 & 72 & 51 & 37 & 36 \\
\hline $\begin{array}{l}\text { Tension } \\
\text { vapeur d'eau }\end{array}$ & 9,37 & $\mathrm{~B}, 55$ & 9,07 & 18,73 & 24,44 & 27,41 & 27,97 & 28,07 & 28,84 & 21,15 & 12,96 & 10,42 \\
\hline
\end{tabular}

\section{d) Insolation}

Le nombre moyen d'heures d'insolation a été calculé pour la période 1965-1967; les moyennes mensuelles sont présentées dans le tableau $\mathrm{n}^{\text {10 }}$ III.

\section{e) Evaporation}

Le total annuel de l'évaporation évalué sui- vant différentes méthodes, est compris entre $2.290 \mathrm{~mm}$ et $3.778 \mathrm{~mm}$. Le maximum se situe en mars, avec $367 \mathrm{~mm}$.

\section{f) Pluviométrie}

Les hauteurs mensuelles de précipitations maximales et minimales observées pour une période de 25 ans sont résumées dans le tableau $n^{\circ} I V$, ainsi que leur moyenne.

TABLEAU $\mathbb{N}^{\circ} I V$

Précipitations mensuelles dans la région du lac Tchad.

\begin{tabular}{|c|c|c|c|c|c|c|c|c|c|c|c|c|c|}
\hline Mois & J & F & M & A & M & J & J & A & S & 0 & N & D & Tota1 \\
\hline $\begin{array}{c}\text { Maximum } \\
\text { observế }\end{array}$ & 0 & 0 & 0 & 0 & 0 & traces & 91,7 & 331,2 & 97,7 & & 0 & 0 & 520,6 \\
\hline $\begin{array}{c}\text { Minimum } \\
\text { observé }\end{array}$ & 0 & 0 & 0 & 0 & 23,1 & 3,0 & & 56,0 & 10,7 & 0 & 0 & 0 & 92,8 \\
\hline Moyenne & 0 & traces & Lraces & 0,7 & 5,5 & 9 & 67,4 & 171,9 & 47 & 6,4 & 0 & 0 & 307,9 \\
\hline
\end{tabular}

Les précipitations sont rigoureusement nulles pendant 6 mois (novembre-avril); elles sont maximales en août $(142 \mathrm{~mm})$. Les mois de juillet, août et septembre voient tomber 90 p. 100 des précipitations annuelles.

Les hauteurs annuelles de précipitations observées pendant 25 ans ont pour maximum $520,6 \mathrm{~mm}$, pour minimum $92,8 \mathrm{~mm}$, et pour médiane $307,9 \mathrm{~mm}$.

La hauteur moyenne interannuelle est de $303 \mathrm{~mm}$; elle atteint $190 \mathrm{~mm}$ pour les décennales sèches et $500 \mathrm{~mm}$ pour les décennales humides. Il y a en moyenne 25,2 jours de pluie.

\section{B. Géologie}

Excepté quelques formations à faciès sidérolithique reposant sur le socle, le remplissage du bassin est de type fluviolacustre avec tous les intermédiaires entre l'argile pure et les sables pluviatiles modelés en ergs fixés.

\section{Végétation}

\section{a) Pâturages naturels}

Le lac Tchad présente un type particulier de végétation, il s'agit d'une végétation aquatique liée au milieu.

Les meilleurs pâturages se trouvent sur les îles les plus basses, aplanies par l'érosion, leur altitude est de 8 à 12 mètres au-dessus des eaux à l'Est et elle s'abaisse vers l'Ouest.

La végétation des rives nigérianes du lac Tchad a été décrite par GOLDING et GWYNNE (1939), KONE (1948) (8) et GASTON (1967) (4) a étudié celle de la rive tchadienne et des îles sableuses.

Selon ce dernier, la végétation aquatique conditionnée par l'eau du lac est importante au point de vue pâturage.

Cet autcur distinguc:

1. les dépressions temporairement inondées ap- 
TABLEAU $N^{\circ}$ III

Insolations moyennes dans la région du lac Tchad.

\begin{tabular}{|c|c|c|c|c|c|c|c|c|c|c|c|c|c|}
\hline Mois & $\mathrm{J}$ & $F$ & $M$ & A & M & $\mathrm{J}$ & $\mathrm{J}$ & A & $\mathrm{s}$ & 0 & $\mathrm{~N}$ & $\mathrm{D}$ & Total \\
\hline $\begin{array}{l}\text { Nombre d'heures } \\
\text { d'insolation }\end{array}$ & 297,5 & 279,9 & 284,1 & 274,9 & 306,6 & 279,1 & 283,2 & 217,7 & 258,1 & 314,6 & 308,5 & 298,0 & $3.402,2$ \\
\hline $\begin{array}{l}\text { Nombre d'heures } \\
\text { d'insolation } \\
\text { théorique }\end{array}$ & 336,2 & 322,8 & 358,9 & 356,8 & 378,0 & 370,2 & 381,1 & 373,9 & 352,7 & 351,9 & 328,1 & 333,8 & \\
\hline $\begin{array}{l}\text { Insolation } \\
\text { relative }\end{array}$ & 0,884 & 0,895 & 0,792 & 0,770 & 0,811 & 0,754 & 0,743 & 0,582 & 0,732 & 0,894 & 0,940 & 0,893 & \\
\hline
\end{tabular}


partenant au système des bras du lac. Ce sont de larges ouaddis inarbustifs.

La végétation est à Sporobolus helvolus et Sporobolus spicatus;

2. la végétation des rives sableuses crée des zones appétées, le plus souvent composées de : Imperata cylindrica, Cynodon dactylon, Paspalidium geminatum et Panicum subalbidum;

3. la bordure flottante est composée de Phragmites mauritianus, Typha angustifolia, Cyperus papyrus et Pennisetum sp.

Parmi ces herbes on rencontre: Oxystelma bornouense et Ludwigia leptocarpa, une fougère: Cyclosorus gongylodes et des Cucurbitaceae comme Mukia maderaspatana.

Cette bordure flottante n'est pratiquement pas touchée par les animaux, de même que les îlots bancs et les îles flottantes. Ces formations sont des masses végétales fixes ou mobiles composées essentiellement de Cyperus papyrus.

Sur le plateau continental, les pâturages sont du type sahélien où l'on rencontre des andropogonées, des graminées annuelles (Aristida mutabilis, Cenchrus biflorus, Eragrostis tremula) et des mimosées.

Sur les îles surélevées, la flore est de composition identique à celle du littoral.

Sur les îles plates, le tapis herbacé est constitué par des andropogonées vivaces à rhizomes, et sur lequel s'implantent des acacias rabougris.

\section{b) Rendement et charge}

D'après GASTON, les dépressions temporairement inondées, qui appartiennent au système des bras du lac, ont une production de matière verte importante par rapport aux dunes environnantes grâce aux Sporobolus vivaces.

De même, la frange de bordure, bien que de largeur réduite (20 mètres environ) amène une production de matière verte importante et ce tout le long de l'année. La longueur des rivages du lac et des îles fait que cette production n'est pas négligeable et intervient pour une grande partie dans l'alimentation des troupeaux sédentaires de la région.

La charge des îles et presqu'îles du lac est faible, le parcours très fréquenté laisse une surface importante du sol nu, les espèces vivaces ont disparu, sauf quelques rares touffes.
On peut compter une tête pour 10 hectares. De plus, la végétation naturelle est sur le sable de plus en plus remplacée par des cultures.

La production annuelle des franges appétées, difficilement chiffrable, est de l'ordre de 2 à 5 tonnes/hectare de matière verte par an.

Les troupeaux des rives du lac complètent là leur ration en lest et en matières protéiques, ce qui leur permet de franchir la période difficile de la saison sèche chaude.

\section{MORPHOLOGIE}

\section{Caractéristiques générales}

Les caractères ethniques de la race ont été décrits par MALBRANT et collab. (1947) (11), DOUTRESSOULLE (1947) (3), KONE (1948) (8) et LAPLANCHE (1969) (10).

D'allure générale imposante, l'animal massif, à ossature développée, offre un aspect lourd, mou, lymphatique et se caractérise par un cornage énorme mais léger, typique de la race, et par l'absence de bosse.

Ce taurin est un animal rectiligne, longiligne, de grande tuille : 140 à $150 \mathrm{~cm}$ (certains animaux âgés atteindraient 170 à $180 \mathrm{~cm}$, et de robe claire (blanche ou isabelle).

Solidement charpenté, ce bœuf atteint un poids moyen de $550 \mathrm{~kg}$, et dans certaines conditions d'engraissement, 600 à $700 \mathrm{~kg}$.

Le dimorphisme sexuel statural est très accusé.

\section{Caractères ethniques}

\section{a) Aspect général}

La tête est longue, large et épaisse, avec un chignon très important, à crête supérieure déprimée en forme de V. I e front est large, avec des arcades orbitaires saillantes.

Le profil est rectiligne, long et bien proportionné à la masse céphalique.

Le mufle est haut et large.

Les oreilles longues et larges, ont un port horizontal. 


\section{b) Les cornes}

Les cornes sont caractéristiques: à l'état normal, elles ont de 70 à $130 \mathrm{~cm}$ de longueur avec 20 à $30 \mathrm{~cm}$ de circonférence à la base. De couleur claire (blanc jaunâtre, grise ou blanche), à pointes noires, elles s'élèvent soit en haute lyre, soit en croissant largement ouvert, la pointe dirigée en arrière.

Ces cornes, énormes mais légères, très spectaculaires, donnent à la tête de l'animal une expression de lourdeur, lourdeur apparente, puisque la cheville osseuse, poreuse et globuleuse est constituée d'aréoles à parois minces, le revêtement corné étant lui-même peu épais.

Les cornages normaux s'observent surtout sur les troupeaux de terre ferme; dans les régions insulaires, on observe une grande variété de cornes.

Celles-ci peuvent prendre des proportions considérables : 130 à $150 \mathrm{~cm}$ de long, et 80 à $100 \mathrm{~cm}$ de circonférence; parfois, ces cornes sont courtes $(15$ à $25 \mathrm{~cm})$ et de diamètre impor-tant.

Les cornes les plus curieuses sont les cornages en bouée (cornes boursouflées, en forme d'oignon), en oreille (cornes aplaties, rugueuses et striées), en courge.

Les cornes mobiles, dites tombantes et flottantes, ou réduites à un moignon, ne sont pas exceptionnelles, certains sujets sont même dépourvus de cornes.

Contrairement à une opinion largement répandue, le cornage des Kouris n'assure pas la flottaison de la tête lors des traversées à la nage des chenaux du lac Tchad.

Ces animaux nagent en maintenant leur cornage hors de l'eau; certains taurins porteurs de cornes volumineuses, sont même mauvais nageurs et, pour empêcher leur noyade pendant la traversée entre les îles, les éleveurs fixent à la base de leur queue un tronçon de bois d'ambadj (Herminiera claphroxylon); par con-

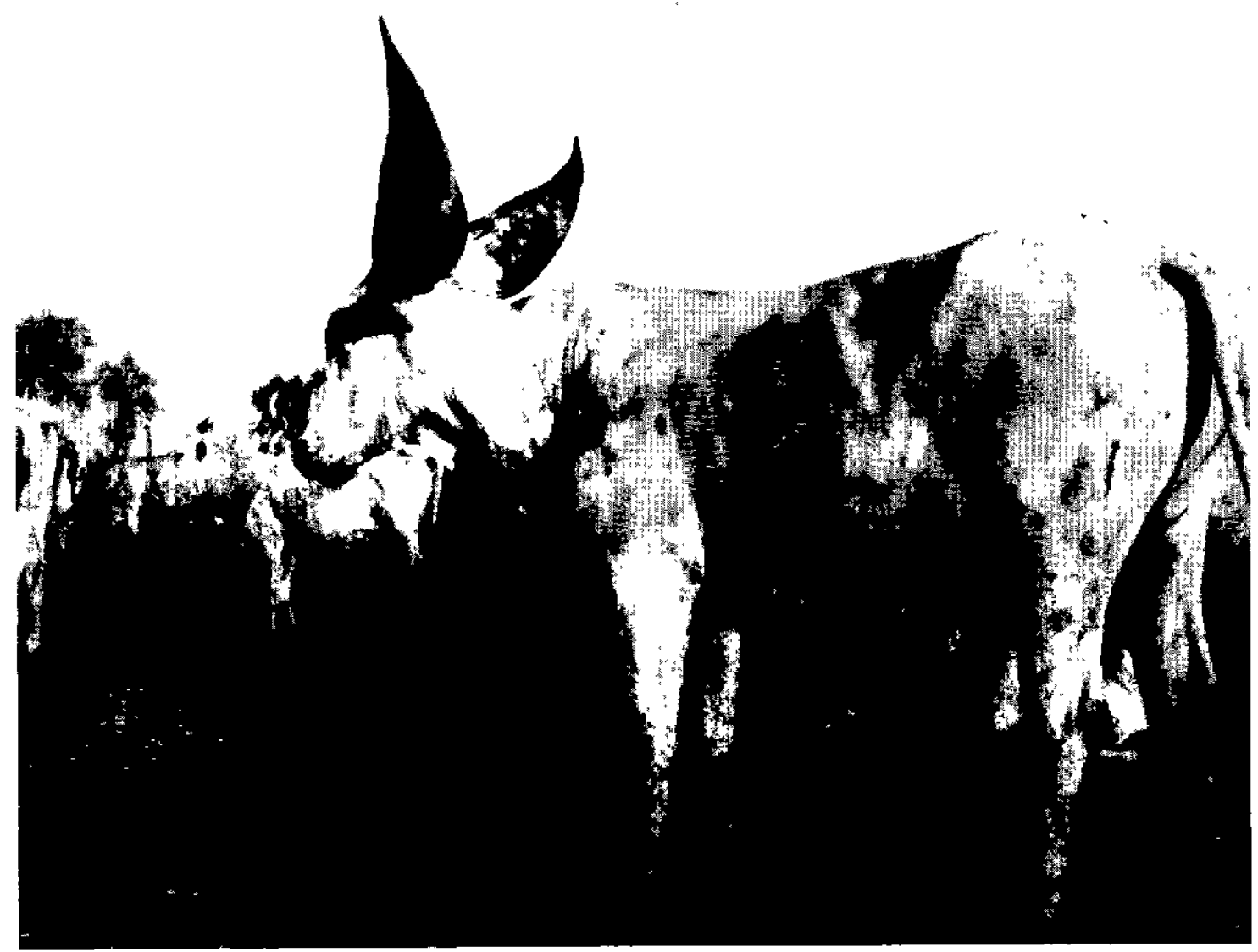

Photo $n^{0}$ 2. - Bouf Kouri à cornage caractéristique de la race. 


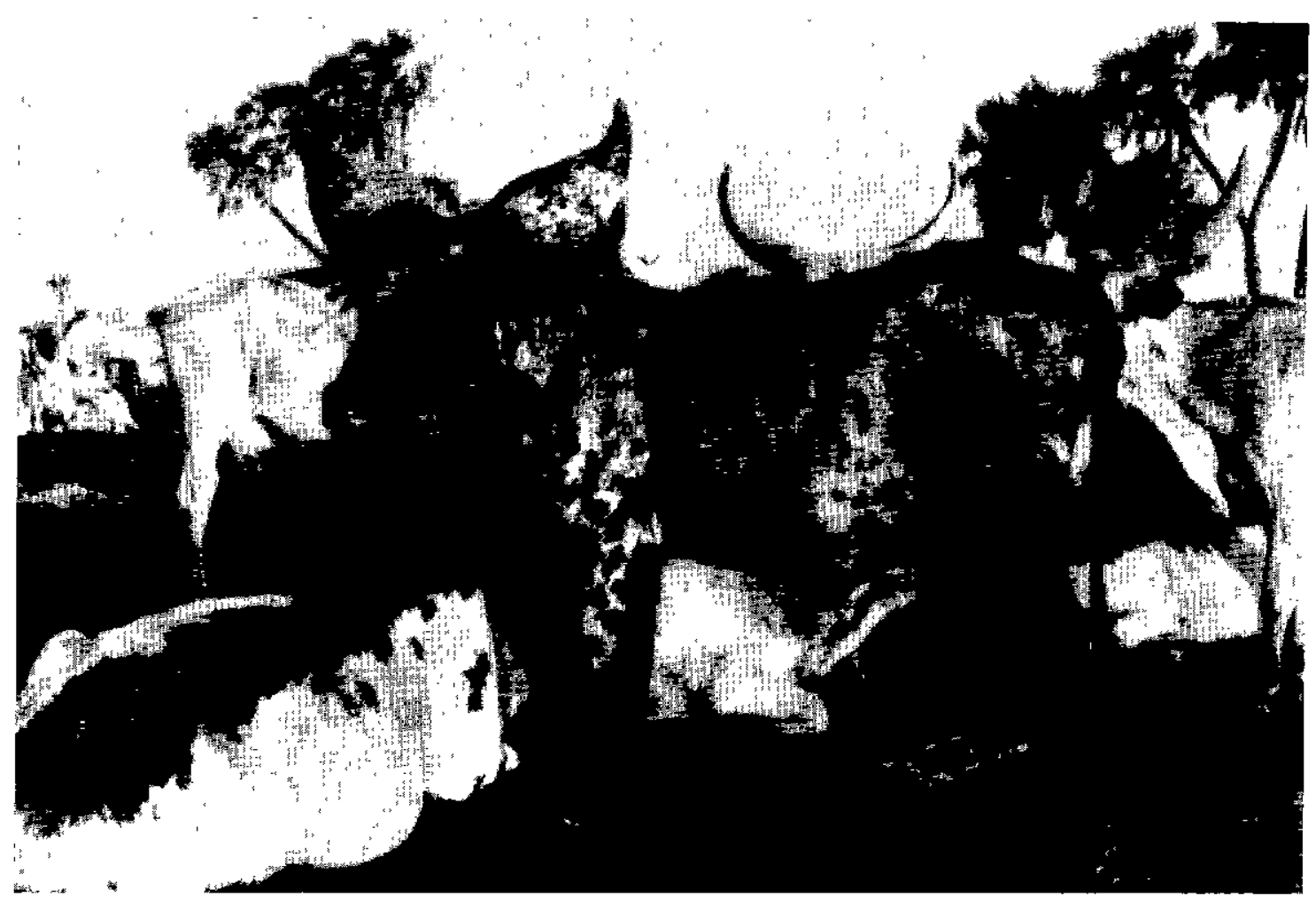

Photo no 3. - Un type de cornes en bouée chez un Kouri dont la robe n'est pas caractéristique.

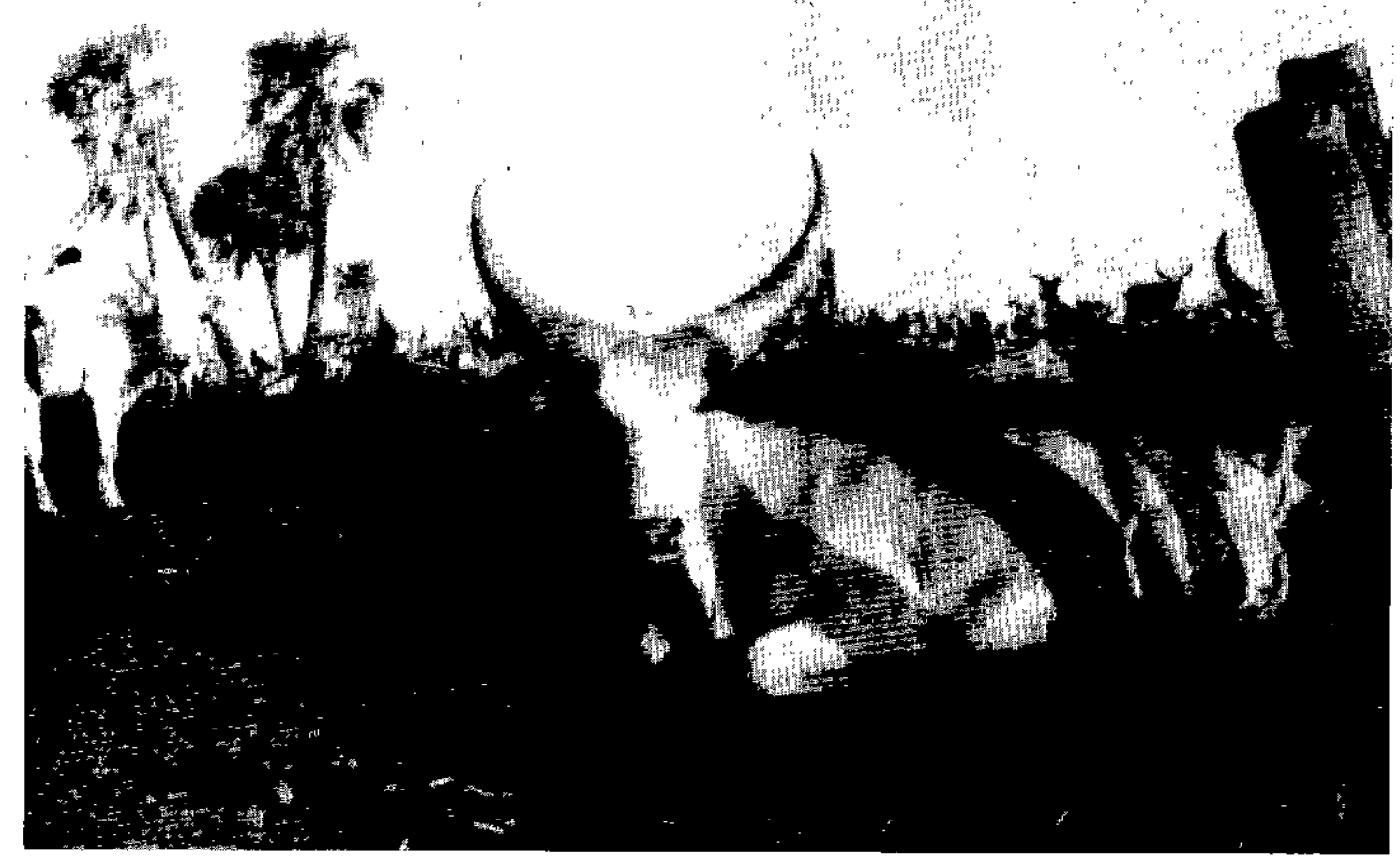

Photo $n^{\circ}$ 4. - Dans un troupeau, un Kouri avec cornage en lyre. 


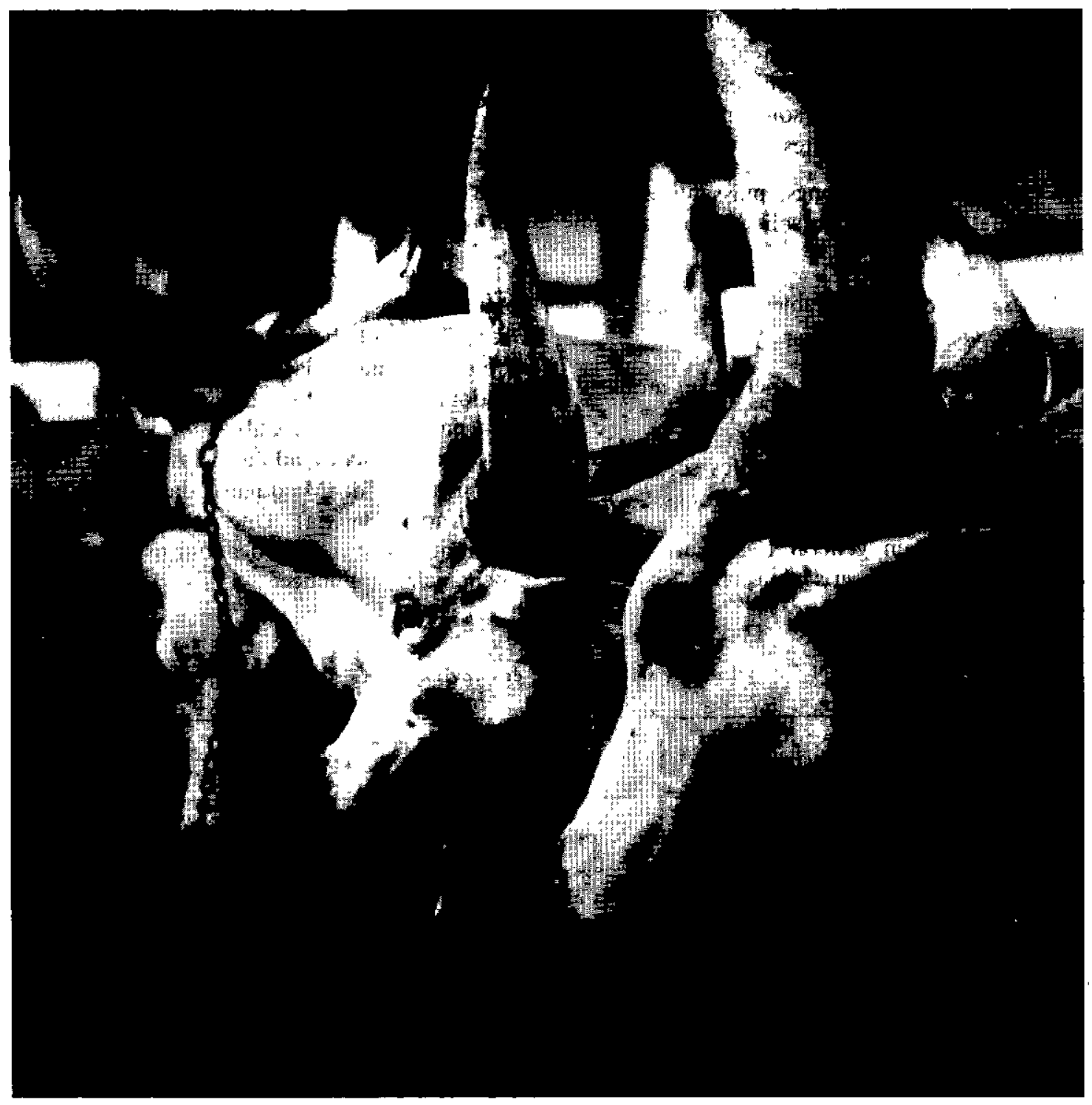

Photo $\mathrm{n}^{\circ}$ 5. - - Une étable de Kouris à cornage en lyre.

tre, des sujets acéres ou à cornes normales nagent aisément.

Les éleveurs Boudoumas préfèrent les animaux porteurs d'un cornage en lyre ou en croissant parce qu'ils prétendent que les sujets présentant un cornage en bouée ont une longévité moindre.
En outre, les vaches acéres ou à cornes flottantes ont la réputation d'être meilleures laitières.

c) Description des autres caractères ethniques

L'encolure est courte et plate, mince chez la vache, assez épaisse chez le bœuf, épaisse 
chez le taureau. Le garrot empâté est exempt de bosse, le fanon extrêmement réduit et l'épaule longue et bien plaquée.

La poitrine est haute, longue et profonde.

Le corps est long et le dos droit. Les hanches sont arrondies, le bassin long et large. La queue implantée haut, longue, assez épaisse, à toupillon long et bien fourni, est bien plaquée entre les fesses. Celles-ci sont droites et larges, les cuisses longues, plates et bien musclées.

Les membres sont longs, puissants et épais, à articulations larges et solides, aux aplombs réguliers.

Les sabots clairs et friables sont larges, épais et très ouverts.

La peau est assez fine, onctueuse, mobile, à poils ras.

Chez les femelles, les mamelles sont rondes, bien développées, et les trayons longs.

La robe est en général très claire, uniformément blanche ou gris clair chez les Kouris de race pure; quelquefois mouchetée de noir ou de noir grisâtre (pourtour des oreilles, tête, cou, plus rarement : poitrail et partie antérieure de la poitrine).

Le mufle, le dessous de la langue, les paupières et l'intérieur des oreilles sont de pigmentation foncée ou noire.

Les données pondérales et les mensurations moyennes relevées dans la littérature sont rares, fragmentaires et reflètent mal les paramètres standards biométriques de cette population taurine.

Le tableau $\mathrm{V}$ résume les mensurations moyennes des Kouris rapportées par MALBRANT et collab. pour des taureaux, vaches et bœufs adultes; par KONE pour 78 bœufs Boudoumas et de tout âge et de tous sexes; enfin par JOSHI et collab. (1957) (7) pour les taureaux et vaches adultes élevés à la ferme administrative de Maiduguri, dans la province du Bornou (Nigéria). Une étude relative aux mensurations et indices des taurins Kouris sera prochainement publiée.

TABLEAU $\mathrm{N}^{\circ} \mathrm{V}$

Mensurations moyennes des bovins Kouris

\begin{tabular}{|l|c|c|c|c|c|c|}
\hline \multirow{2}{*}{ Nature des mensurations } & \multicolumn{5}{|c|}{ A u t e u $\mathrm{r} \mathrm{s}$} \\
\cline { 2 - 6 } & \multicolumn{3}{|c|}{ Malbrant et Collab., } & \multicolumn{2}{c|}{ Joshi et Collab., } & Kone \\
\hline & Taureaux & Vaches & Boeufs & Taureaux & Vaches & \\
Poids en kg & 650 & 400 & 550 & 500 & 360 & 480 \\
Hauteur de la croupe (en cm) & 154,6 & 145,4 & 156,6 & - & - & 158 \\
Hauteur au garot (en cm) & 146,0 & 136,0 & 151,0 & 152 & 140 & 149 \\
Hauteur au passage des sangles (en cm) & 77,2 & 69,0 & 78,1 & - & - & - \\
Largeur des hanches (en cm) & 49,2 & 46,0 & 50,2 & 48 & 45 & 34 \\
Longueur scapulo ischiale (en cm) & 122,3 & 116,3 & 124,0 & 152 & 144 & 165 \\
Périmètre thoracique (en cm) & 195 & 184 & 211 & 193 & 172 & 183 \\
Longueur de la tête (en cm) & 65,9 & 59,3 & 68,0 & - & - & 61 \\
Largeur de la tête (en cm) & 32,1 & 24,6 & 27,5 & - & - & - \\
\hline
\end{tabular}

En conclusion, le bœuf Kouri est un animal sans bosse, à robe blanche, au cornage caractéristique, de grande taille, aux membres bien développés, à la silhouette massive, de caractère placide.

\section{APTITUDES}

Le Kouri est un animal rustique, mou, lymphatique et doux, parfaitement adapté à son milieu. Les performances du bétail Kouri ne sont connues qu'imparfaitement.

Les caractéristiques de croissance, de précocité, de fécondité, de productivité de la race sont estimées mais les limites potentielles ne sont pas connues.

\section{a) Travail}

Le dressage des animaux n'a d'autre but que le portage. Cette aptitude au portage est 
médiocre chez cet animal aux allures lentes dues à son tempérament lourd et lymphatique.

Les bœufs du lac Tchad se déplacent d'île en île et nagent facilement.

\section{b) Croissance}

Dans la littérature, le poids moyen à la naissance serait de l'ordre de $25 \mathrm{~kg}$ pour les veaux mâles, et de $22,5 \mathrm{~kg}$ pour les veaux femelles. On estime que le développement d'adulte est atteint à 5 ans.

\section{c) Précocité sexuelle et fécondité}

La race semble relativement précoce : d'après RECEVEUR, la première mise bas peut intervenir à 3 ans, mais l'âge moyen du premier vêlage est compris entre 3 ans et demi et 4 ans; la saillie a donc lieu entre 2 ans et 9 mois et 3 ans et 3 mois.

Les intervalles entre les vêlages successifs sont en moyenne de 15 à 18 mois.

La vie sexuelle active des femelles s'étend entre 11 et 12 ans et le nombre de vêlages se chiffre entre 6 et 8 .

D'après les données recueillies auprès des éleveurs, le nombre de veaux par femelle se répartirait de la façon suivante:

- 2 à 3 veaux: 20 p. 100

- 4 à 6 veaux: 40 p. 100

-7 à 8 veaux: 30 p. 100

-9 veaux et plus : 10 p. 100

Le nombre de femelles stériles serait de l'ordre de 3 p. 100.

\section{d) Productivité}

\section{Sécrétion lactée}

L'aptitude laitière semble assez développée comparativement aux autres races bovines africaines. Le rendement quotidien d'une bonne vache en pleine lactation serait compris en moyenne entre 3 et 6 litres. contenant 30 à 35 grammes de matières grasses par litre. La durée des lactations serait de 6 à 10 mois.

En général, la durée de la lactation varie avec son numéro ( $1^{\text {re }}, 2^{\mathrm{e}}$ et $n^{\mathrm{e}}$ lactation) et avec l'époque du vêlage.

Lors du premier vêlage, la lactation est insuffisante; les deuxième, troisième et quatrième vêlages offrent une bonne lactation; enfin, pour les vêlages ultérieurs, la sécrétion lactée décroît progressivement.

Les lactations obtenues de juillet à octobre sont plus abondantes que celles de même ordre obtenues en dehors de cette période.

Le sevrage du veau correspond au tarissement de la sécrétion lactée. Les vaches subissent une traite quotidienne de décembre à mai et deux de juillet à novembre.

La traite est effectuée à la pincée en présence du veau. Les dernières portions sont impossibles à obtenir à la main et sont laissées au veau. Le lait (frais ou caillé) est consommé par les éleveurs, ou utilisé pour la production de beurre.

\section{Production de viande}

Prêts à l'abattage vers 5 ans, les Kouris sont parmi les meilleurs animaux de boucherie que l'on puisse trouver en zone sahélienne.

Leur carcasse présente une proportion de morceaux de première catégorie supérieure à la moyenne (RECEVEUR).

Les opinions divergent quant à la proportion d'os :

- faible (18 p. 100 - RECEVEUR);

- élevée (JOSHI).

Le rendement est de l'ordre de 50 p. 100 en viande nette.

La viande tendre, savoureuse, est persillée, fait exceptionnel en région tropicale.

D'après les données de LACHAUX et collab. (1968) (9) et MARTIN (1969) (12), il a été calculé qu'à l'abattoir frigorifique de Farcha - Fort-Lamy, de début 1967 à fin 1968, sur 83.771 carcasses, le bœuf du lac intervient pour 2.582 têtes, soit 3,0 p. 100 , la répartition par sexe étant la suivante :

— animaux castrés: 1.564 , soit 60,5 p. 100 ;

- animaux mâles: 945 , soit 36,5 p. 100 ;

— animaux femelles: 73, soit 3,0 p. 100.

Dans les tableaux VI et VII figurent les résultats des abattages des animaux de race Kouri (nombre, poids total et moyen) par sexe et par âge (les statistiques sur les poids n'ont été effectuées que tous les deux mois). 
TABLEAU $\mathrm{N}^{\circ} \mathrm{VI}$

Statistiques des abattoirs de Fort-Lamy concernant l'abattage des Kouris castrês, mâles et femelles.

\begin{tabular}{|c|c|c|c|c|c|c|c|c|}
\hline \multirow[b]{2}{*}{ Sexe } & \multirow{2}{*}{$\begin{array}{r}\text { Nature de 1a } \\
\text { statistique }\end{array}$} & \multicolumn{6}{|c|}{$A g$ e $s$} & \multirow[b]{2}{*}{ Total } \\
\hline & & 4 ans & 5 ans & 6 ans & 7 ans & 8 ans & $\begin{array}{l}9 \text { uns } \\
\text { et plus }\end{array}$ & \\
\hline \multirow{3}{*}{ Castrës } & Nombre & 209 & 232 & 107 & 134 & 54 & $1 B$ & 754 \\
\hline & Poids total en $\mathrm{kg}$ & 36.390 & 42.880 & 21.383 & 29.629 & 11.382 & 4.217 & 145.881 \\
\hline & Poids moyen en $\mathrm{kg}$ & 174,1 & 164,8 & 199,5 & 221,1 & 210,7 & 234.3 & 193,4 \\
\hline \multirow{3}{*}{ Mâles } & Nombre & 162 & 94 & 43 & 58 & 17 & 1 & 375 \\
\hline & Poids total en $\mathrm{kg}$ & 26.217 & 16.818 & 8.288 & 11.786 & 3.680 & 180 & 66.969 \\
\hline & Poids moyen en $\mathrm{kg}$ & 161,8 & 178,8 & 192,7 & 203,2 & 216,4 & 180 & 178,3 \\
\hline \multirow{3}{*}{ Femelles } & Nombre & 2 & 4 & 4 & 3 & 10 & 12 & 35 \\
\hline & Poids total en $\mathrm{kg}$ & 288 & 575 & 585 & 511 & 1.691 & 1.928 & 5.578 \\
\hline & Poids moyen en $\mathrm{kg}$ & 144 & 143,7 & 146,2 & 170,3 & 269,1 & 160,6 & 159,3 \\
\hline
\end{tabular}

On remarquera que les poids observés à l'abattoir sont inférieurs, si on les transforme en $\mathrm{kg}$ de poids vifs aux poids rapportés dans le tableau $\mathrm{n}^{\circ} \mathrm{V}$.

TABLEAU Na VII

Résultats globaux concernant les poids des Kouris abattus à Fort-Lamy

\begin{tabular}{|c|c|}
\hline Nombre d'animaux & 1.164 \\
\hline $\begin{array}{c}\text { Poids total } \\
\text { au kg }\end{array}$ & 218.428 \\
\hline $\begin{array}{c}\text { Poids moyen } \\
\text { au kg }\end{array}$ & $\mathbf{1 8 7 , 6}$ \\
\hline
\end{tabular}

Les pourcentages d'abattage, calculés en poids, par sexe et par âge, pour l'ensemble mâles et castrés (97 p. 100) s'établissent comme suit chez les Kouris (tableaux $\mathrm{n}^{\text {us }}$ VIII et IX).

TABLEAU No VIII

Répartition par sexe

\begin{tabular}{|c|c|c|}
\hline Castrés & Mâles & Femelles \\
\hline 66,7 p. 100 & 30,6 p. 100 & 2,5 p. 100 \\
\hline
\end{tabular}

TABLEAU No IX

Répartition par âge (mâles et castrés)

\begin{tabular}{|c|c|c|c|c|c|c|}
\hline Age & 4 ans & 5 ans & 6 ans & 7 ans & 8 ans & $\begin{array}{c}9 \text { ans } \\
\text { et plus }\end{array}$ \\
\hline p. 100 & 29,4 & 28,0 & 13,9 & 19,4 & 7,0 & 2,1 \\
\hline
\end{tabular}

Les Kouris représentent 4 p. $100 \mathrm{du}$ tonnage exporté.

Les carcasses Kouris sont exportées à 81 p. 100 et 19 p. 100 sont consommées sur le marché local.

Les qualités de viande, selon la législation tchadienne, sont: viande de qualité prime,
A et B, que LEVIF définit ainsi: viande de qualité prime : animaux de 4 à 6 ans en très bon état d'engraissement.

- Pour les arabes et les Kouris :

- mâles de 4 ans de $180 \mathrm{~kg}$ et plus;

— mâles de 5 à 6 ans de $200 \mathrm{~kg}$ et plus;

— femelles de 4 à 6 ans de $160 \mathrm{~kg}$ et plus. 


\section{- Pour les Bororos:}

- mâles de 4 ans de $200 \mathrm{~kg}$ et plus;

- mâles de 5 et 6 ans de $220 \mathrm{~kg}$ et plus;

— femelles de 4 à 6 ans de $180 \mathrm{~kg}$ et plus.

Viande de qualité A; animaux de 4 à 8 ans en bon état d'engraissement:

- Pour les arabes et les Kouris :

- mâles de 4 ans de 160 à $180 \mathrm{~kg}$;

- mâles de 5 et 6 ans de 180 à $200 \mathrm{~kg}$;

- mâles de 7 et 8 ans de $180 \mathrm{~kg}$ et plus;
— femelles de 7 et 8 ans de plus de $160 \mathrm{~kg}$.

- Pour les Bororos:

- mâles de 4 ans de 180 à $200 \mathrm{~kg}$;

- mâles de 5 et 6 ans de 200 à $220 \mathrm{~kg}$;

- mâles de 7 et 8 ans de plus de $200 \mathrm{~kg}$;

- femelles de 7 et 8 ans de plus de $180 \mathrm{~kg}$.

Viande de qualité $\mathbf{B}$ : reliquat.

D'après cette classification, et pour les bovins abattus en 1967, la ventilation est la suivante, présentée dans le tableau $\mathrm{n}^{\circ} \mathrm{X}$.

TABLEAU $\mathrm{N}^{\circ} \mathrm{X}$

Répartition des qualitês selon les races aux abat toirs de Fort-Lamy.

\begin{tabular}{|l|c|c|c|}
\hline Qualités & $\begin{array}{c}\text { Pourcentage } \\
\text { chez le Kouri }\end{array}$ & $\begin{array}{c}\text { Pourcentsge } \\
\text { chez le Bororo }\end{array}$ & $\begin{array}{c}\text { Pourcentage } \\
\text { chez l'Arabe }\end{array}$ \\
\hline Viande de qualité prime & $25,6 \mathrm{p} \cdot 100$ & $16,6 \mathrm{p} \cdot 100$ & $10,1 \mathrm{p} \cdot 100$ \\
Viande de qualité A & $35,4 \mathrm{p} .100$ & $39,6 \mathrm{p} \cdot 100$ & $22,1 \mathrm{p} \cdot 100$ \\
Viande de qualité B & $39,0 \mathrm{p} \cdot 100$ & $43,7 \mathrm{p} \cdot 100$ & $67,6 \mathrm{p} \cdot 100$ \\
\hline
\end{tabular}

$\mathrm{Au}$ contraire, la classification des carcasses suivants pour l'année 1968 (tableau $\mathrm{n}^{\circ} \mathrm{XI}$ ). par tranche de poids aboutit aux pourcentages

TABLEAU $N^{*} \mathrm{XI}$

Répartition du poids des carcasses selon les races aux abattoirs de Fort-Lamy.

\begin{tabular}{|l|c|c|c|}
\hline Carcasses de : & $\begin{array}{c}\text { Pourcentage } \\
\text { chez le Kouri }\end{array}$ & $\begin{array}{c}\text { Pourcentage } \\
\text { chez le Bororo }\end{array}$ & $\begin{array}{c}\text { Pourcentage } \\
\text { chez l'Arabe }\end{array}$ \\
\hline P1us de $200 \mathrm{~kg}$ & $30,9 \mathrm{p} .100$ & $68,0 \mathrm{p} .100$ & $11,2 \mathrm{p} \cdot 100$ \\
180 à $200 \mathrm{~kg}$ & $28,1 "$ & $18,1 " 15,0$ & 11 \\
Moins de $180 \mathrm{~kg}$ & $40,9 \mathrm{Nr}$ & $13,7 "$ & 73,7 \\
\hline
\end{tabular}

Les pourcentages d'abattages de Kouris pen- figurent dans le tableau $n^{0}$ XII. dant 2 ans, calculés par tranche de 2 mois

TABLEAU $N^{\circ} X I I$

Apports et répartitions des abattages annuels (p.100) de Kouris à Fort-Lamy.

\begin{tabular}{|c|c|c|c|c|c|}
\hline $\begin{array}{l}\text { Janvier } \\
\text { Février }\end{array}$ & $\begin{array}{l}\text { Mars } \\
\text { Avril }\end{array}$ & $\begin{array}{l}\text { Mai } \\
\text { Juin }\end{array}$ & $\begin{array}{l}\text { Juillet } \\
\text { Août }\end{array}$ & $\begin{array}{l}\text { Septembre } \\
\text { 0ctobre }\end{array}$ & $\begin{array}{c}\text { Novembre } \\
\text { Décembre }\end{array}$ \\
\hline 19,9 & 29,0 & 37,9 & 6,9 & 2,2 & 3,7 \\
\hline
\end{tabular}




\section{MODE D'ELEVAGE}

A l'origine la race autochtone, pure, dominante, a été le Kouri, grand bœuf blanc, sans bosse, à cornes bulbeuses, globuleuses, dont l'élevage se limitait strictement aux îles et berges de l'est du lac Tchad, à la province du Bornou au Nigéría et à la région de N'Guigmi au Niger.

L'élevage de ce taurin se pratique surtout au Tchad, dans la préfecture du Lac, sur le mode extensif, par les tribus Boudouma, Kouri, Kanembou et Keleoua, originaires de Libye et du Soudan, dont il constitue l'une des principales ressources. Son exploitation ne diffère guère de celle de l'élevage traditionnel africain.

Aujourd'hui, on ne rencontre pratiquement plus d'animaux au cornage en bouée, à l'exception de quelques spécimens dans le nord de la zone, à l'intérieur de l'archipel.

De leur berceau d'origine, ces taurins se sont éparpillés dans toutes les directions sauf vers le Nord. Les transactions commerciales ont élargi à l'Est, à l'Ouest et au Sud-Ouest leur zone de dispersion. On remarque souvent des Kouris dans les troupeaux itinérants qui arrivent dans les zones côtières pour être abattus.

D'une manière générale, cette race Kouri est en voie d'absorption par les races de zébus autochtones et ce métissage progressif conduira à sa disparition si des mesures appropriées ne sont pas envisagées.

Ainsi, dans tout le Kanem, voire plus au Sud jusqu'à Fort-Lamy, on peut rencontrer des produits de croisement avec le zébu arabe.

A l'Ouest, la race se rencontre jusque dans la région de Tassout où des sujets sont métissés avec les races Peuhles ou Bororodji; dans ce cas le cornage caractéristique est remplacé par un cornage normal avec la même direction générale, mais de format réduit.

Ces caractères de métissage sont extrêmement variables suivant la région et le sang dominant, le degré de sang taurin diminuant graduellement à mesure que l'on s'éloigne du lac.

Plusieurs causes sont à la source de ce métissage. En 1955-1956, les crues du Logone et du Chari ont provoqué l'inondation des îles basses et de l'archipel du lac, d'où un manque de pâturages et une mortalité dans le cheptel. Pour parer à cette perte, les éleveurs ont croisé leurs Kouris avec des animaux plus rustiques ou ont acheté ces derniers pour reconstituer leurs troupeaux.

Les premiers métissages ont eu lieu dans la zone littorale, principalement dans les cantons de Liwa et d'Isseirom, où entre les éleveurs Fellata et Kanembou d'une part, et les gens du lac (Boudouma et Kouri) d'autre part, existe un mode d'échange de taurillons contre génisses.

En effet, le bétail Kouri étant très coté sur les marchés locaux, les éleveurs Boudouma ou Kouri échangent un mâle adulte contre un, deux ou trois jeunes de race arabe ou foulbé, afin d'accroître numériquement leurs troupeaux plus rapidement.

En outre, ces échanges sont dictés à l'éleveur Kouri par un intérêt zootechnique : les métis de première génération, croisés avec un taureau Kouri pur sang, donnent, selon eux, des produits où les femelles offrent une meilleure lactation et un taux de fécondité amélioré.

Lors de transactions commerciales, les boufs arabes ou foulbés sont préférés par les éleveurs Boudoumas ou Kouris, car leurs prix sont plus bas que ceux pratiqués pour le bétail Kouri.

Cependant, si les éleveurs autochtones dans leurs échanges ou achats portent leur choix sur des races continentales, ils préfèrent garder comme reproducteurs des taurillons typiques de la race Kouri qu'ils jugent mieux proportionnés, mieux conformés, et dont ils apprécient les femelles meilleures laitières.

On peut distinguer deux grandes zones d'élevage :

- la région de Bol comprenant la zone littorale du lac, les îles des cantons de Bol et de N'Guéléa, les cantons de Liwa, Limboy et Tatavérom. Dans cette aire géographique, il n'y a pratiquement pas de transhumance, chaque communauté d'éleveurs possédant un petit nombre d'îles ou une portion du littoral sur lesquels s'effectuent des rotations irrégulières;

- les centres de Kangallon et d'Isseirom : dans cette région s'effectue une transhumance 
dans les zones de cultures de Massakory, les éleveurs étant également agriculteurs.

Contrairement aux Bororos nomades accompagnant toujours leur bétail, les éleveurs Boudoumas ou Kouris, préoccupés par la culture ou quelquefois la pêche, laissent leurs troupeaux paître seuls, en liberté, aux pâturages; ceux-ci rejoignent les villages ou les campements en fin de journée.

L'état d'entretien des taurins Kouris est intiment lié au type de saison et aux variations hydrologiques du lac.

A la saison des pluies correspond un maximum de végétation où les animaux ont un excédent de pâturage et prennent du poids.

Pendant la saison fraîche, d'octobre à mars, le bétail conserve le gain de poids acquis pendant la saison des pluies et présente, à cette époque, le meilleur état d'embonpoint.

A noter, qu'à la même saison, les animaux pâturant sur le littoral ont un embonpoint supérieur au bétail entretenu sur les îles ou dans les régions marécageuses.

En pleine saison sèche, les pâturages se dessèchent et atteignent 90 à 95 p. 100 de matière sèche. A la fin de cette saison, les rations d'entretien sont insuffisantes et les animaux maigrissent.

Les crues du lac provoquent l'inondation de la presque totalité des îles basses qui constituent les meilleurs pâturages insulaires. Aussi les troupeaux se réfugient sur les îles hautes, d'où une forte concentration de têtes de bétail par hectare, et un épuisement rapide de pâturages médiocres.

Ces conditions conduisent inéluctablement à l'amaigrissement, au polyparasitisme, et finalement à la cachexie.

Les troupeaux Kouris sont constitués en moyenne d'une trentaine de têtes dont un taureau.

Les géniteurs mâles sont des animaux à robe blanche sélectionnés d'après leur conformation physique ou d'après la qualité laitière quils transmettent à leurs filles (jugée d'après la finesse de la peau et l'abondance du cérumen).

Les taureaux effectuent leur première saillie à l'âge de 4 ans, parfois à 3 ans - 3 ans et demi, et sont réformés entre 9 et 11 ans, plus généralement à 10 ans. Les mâles, non retenus pour la reproduction et destinés à la boucherie, sont castrés à 3 ans, selon une technique traditionnelle ou moderne.

La reproduction s'opère tout au long de l'année, la période de vêlage ayant cependant une plus grande fréquence en décembre-janvier.

\section{EXTENSION DE LA RACE}

La multiplication de la race n'étant pas possible sur l'aire géographique restreinte dans laquelle le Kouri rencontre ses conditions optimales de vie, des tentatives d'introduction de la race ont été essayées en diverses régions du Tchad (Lac Fitri, Bas-Chari, Lac de Fianga), au Niger (région de Tillabery) et au Nigéria (Maiduguri).

En 1948, et jusqu'en 1958, une expérience d'élevage du bouf Kouri, hors de sa zone d'élevage lacustre, a été entreprise à N'Gouri, pour l'étude biométrique de Ieur croissance.

A la ferme administrative de Fianga, dans la région de Mayo-Kebbi, entre 1953 et 1957 , des investigations ont été effectuées sur:

- la transplantation du bœuf du lac Tchad, hors de son habitat naturel;

- la création d'une race métisse par croisement N'Dama $\times$ Kouri, pour la diffusion d'une race laitière trypanotolérante.

La tentative d'acclimatement d'un troupeau Kouri de 70 à 90 têtes n'a pas donné les résultats escomptés; par voie de conséquence, les métis N'Dama $\times$ Kouri n'étaient plus réalisables.

Un troupeau Kouri appartenant à l'Institut de Recherches du coton et des textiles de la station de Tickem vit depuis une vingtaine d'années au bord du lac de Tickem où l'eau et l'herbe sont en abondance.

Les essais effectués au Niger, dans la région de Tillabery, vers les années 1935-1940 ont été abandonnés pour des raisons d'économies. Les animaux s'étaient pourtant bien adaptés.

Au Nigéria, à la ferme administrative de Maiduguri, dans la province du Bornou, un troupeau d'élevage est entretenu et son rendement laitier étudié. Son noyau de reproduc- 
teurs, composé de 10 vaches et d'un taureau fut importé en 1944.

\section{PATHOLOGIE}

\section{Maladies parasitaires internes}

a) Helminthes

Au nord du $13^{e}$ parallèle, la proportion de veaux de lait (jusqu'à 5 mois) parasités représente 17 à 20 p. 100 , sauf au lac Tchad où l'on ne compte que 0,6 p. 100 .

Dans la préfecture du Lac, l'ascaridiase, à un taux insignifiant $(0,6$ p. 100), constitue la seule maladie parasitaire des veaux de lait, alors que partout ailleurs prévaut un polyparasitisme à base de Neoascaris vitulorum, de Strongles divers, surtout Haemoncus et Bunostomes, plus rarement Esophagostomes, des Coccidies et Strongyloides papillosus.

Au lac Tchad, 99 p. 100 des bouvillons sont porteurs de parasites internes.

Les espèces déterminées qui coexistent le plus souvent sont au nombre de 16, chez le bouvillon, et ont pour dominantes des Nématodes: Haemoncus contortus et Haemoncus placei de la caillette (65,3 p. 100) des animaux, Bunostomum phlebotomum (64,6 p. 100), Bosicola radiatum $(63,7$ p. 100$)$, Cooperia punctata et Cooperia pectinata (12 p. 100), Artionema labiato papillosa du péritoine (20 p. 100), Onchocerca armillata (11 p. 100) et Buckleyuris globulosa (2,6 p. 100).

Parmi les Trématodes :

- Fasciola gigantica (16,9 p. 100);

- Paramphistomum microbothrium (16,9 p. 100).

Enfin, les Cestodes rencontrés sont:
- Schistosoma bovis (16,9 p. 100);

- Moniezia expansa (4,4 p. 100);

- Moniezia benedeni (3,7 p. 100);

- Thysanezia ovilla (10 p. 100);

- Carmyerius spatiosus (0,9 p. 100);

- Avitellina centripunctata.

Chez les Kouris adultes, 100 p. 100 des animaux sont parasités. Les affections à Trématodes dominent : Fasciola gigantica des canaux biliaires (91,5 p. 100), Schistosoma bovis (81 p. 100), les Paramphistomidés $(87,3$ p. 100$)$ et les Gastrothylacidae $(8,4$ p. 100$)$.

Parmi les affections à Nématodes, l'œsophagostomose sévit dans la préfecture du Lac à 42 p. 100, alors que dans toutes les autres préfectures du Nord du Tchad, elle n'est en cause que dans moins de 15 p. 100 des cas.

Les filarioses à Artionema et à Onchocerca se rencontrent respectivement chez 41 et 95.7 p. 100 des animaux.

Les autres pourcentages d'infestation sont: - Haemoncus $(68,4$ p. 100$)$,

- Bunostomum phlebotomum (6,3 p. 100),

- Cooperia punctata (5,2 p. 100),

- Cooperia pectinata (14,7 p. 100),

- Buckleyuris globulosa (1 p. 100).

Enfin le téniasis touche 30,5 p. 100 des animaux adultes.

Les saisies, opérées à l'abattoir sur les animaux de race Kouri, montrent chez ces derniers 50 p. 100 de foies à distomatose, alors que les zébus arabes, vivant en zone sahélienne, sont pour la plupart parasités à moins de 10 p. 100 (MARTIN, 1969) (12).

TABLEAU $\mathbb{N}^{\circ} \mathrm{XIII}$

Saisies d'abattoir sur les Kouris à Fort-Lamy.

\begin{tabular}{|c|c|c|c|c|}
\hline $\begin{array}{l}\text { Animaux } \\
\text { abattus }\end{array}$ & $\begin{array}{l}\text { Saigies } \\
\text { totales }\end{array}$ & \multicolumn{3}{|c|}{ Saisies partielles } \\
\hline \multirow{3}{*}{1.503} & \multirow{3}{*}{3} & $\begin{array}{c}\text { Foies à } \\
\text { distomatose }\end{array}$ & $\begin{array}{l}\text { Coeurs à } \\
\text { péricardite }\end{array}$ & $\begin{array}{l}\text { Langues à } \\
\text { cysticercose }\end{array}$ \\
\hline & & 819 & 7 & 5 \\
\hline & & 0,2 p.100 & 54,5 p. 100 & $0,3 \mathrm{p} \cdot 100$ \\
\hline
\end{tabular}




\section{b) Zoonoses parasitaires}

L'échinococcose-hydatidose ne se rencontre pas chez les bouvillons dans la préfecture du Lac et son incidence est très faible chez les adultes $(0,66$ p. 100 des bovins).

Les taux d'infestation relatifs à la cysticercose bovine sont respectivement de 9,7 p. 100 et de 14,7 p. 100 chez les animaux âgés de 6 à 30 mois et les adultes.

\section{c) Protozoaires}

Les hémoparasitoses majeures rencontrées chez le Kouri sont les trypanosomoses et les piroplasmoses.

Piroplasma bigeminum est le plus communément rencontré mais, nécessitant une médecine individuelle, son traitement n'est que pratique.

Dans les statistiques du Service de l'Elevage du Tchad, on relève qu'en 1956 un foyer de trypanosomose provoqua une mortalité de 22,3 p. 100 parmi les Kouris du troupeau zootechnique de la ferme de N'Gouri. En brousse, les chiffres sont beaucoup plus mal connus et l'on remarque des chiffres variant de 50 malades, en 1951, à 7.494, en 1961. En réalité, les éleveurs ont du mal à individualiser cette maladie d'un complexe qu'ils dénomment "Bou ".

Ce terme Kanembou signifie : perte de sang, c'est-à-dire anémie ou bien encore maladie qui entraîne l'amaigrissement, la cachexie et la mort.

Le "Bou" ne représente pas une entité pathologique mais un syndrome clinique dû à l'addition de diverses infestations parasitaires importantes dans ces zones humides qui atteignent chacune à un même moment une acuité certaine.

Ce syndrome sévit chez les bovins au cours de la saison sèche, période de disette, qui s'étend de décembre à juillet, où les animaux séjournent longtemps dans l'eau, traversent d'île en île, à la recherche de pâturages.

Les pertes enregistrées seraient de l'ordre de 4 à 5 p. 100 .

\section{Maladies parasitaires externes}

Les taurins Kouris sont réputés être relativement indifférents aux parasites extérieurs, bien que chaleur et humidité soient défavorables à la pullulation des arthropodes (Tabanidés, Muscidés, Simulidés, Culicidés et Ixodidés).

La population ixodidienne est représentée par :

- Boophilus annulatus (SAY, 1821); l'espèce semble bien établie au pourtour du lac Tchad, surtout abondante dans la zone d'inondation;

- Boophilus decoloratus (KOCH, 1844);

- Hyalomma impeltatum (SCHULZE et SCHLOTTKE, 1930);

- Rhipicephalus sanguineus (LATREILLE, 1806);

- parmi les Argasidae: Ornithodoros savigneji (AUDOIN, 1827).

MOREL et GRABER, (1961) (13) ont rapporté la présence de Rhipicephalus appendiculatus sur les rives du lac Tchad. Il s'agirait d'un cas d'introduction isolé, sans signification réelle, dans la population des tiques de la région.

\section{Maladies infectieuses virales}

Bien que sensible à la pathologie classique africaine, le bétail Kouri ne paie plus de lourds tributs aux maladies infectieuses virales ou microbiennes.

Les campagnes de prophylaxie contre Ia peste bovine, réalisées par le Service de l'Elevage du Tchad à l'aide d'un vaccin de cultures cellulaires, ont conduit à l'élimination des grandes enzooties. Cependant, si une vaccination massive entraîne la diminution du nombre de foyers et de leur agressivité, l’éradication totale et définitive de la peste bovine reste à réaliser.

Dans la préfecture du lac, où l'on estime le cheptel à 300.000 têtes de bétail, 222.541 vaccinations contre la peste bovine, soit 74,1 p. 100 du cheptel, ont été effectuées en 1969.

La maladie des muqueuses, maladie des bovins actuellement extensive au Tchad, se rencontre également dans la région du lac Tchad. L'allure clinique et épizootiologique de la maladie presque identique à celle de la peste bovine, pose un problème quant à l'épidémiologie et la prophylaxie de cette dernière. 


\section{Maladies infectieuses bactériennes}

La péripneumonie contagieuse des bovidés a disparu de la région du lac à la suite de l'application de mesures de prophylaxie médicale et sanitaire. Précisons que la péripneumonie bovine en zone sahélienne présentait des foyers moins nombreux qu'en zone soudanienne.

Les vaccinations contre le charbon symptomatique et le charbon bactéridien ne sont pas systématiques. Quelques foyers sporadiques éclatent au début de la saison des pluies. Les vaccinations pratiquées dans ce cas ne portent que sur $0,8 \mathrm{p} .100 \mathrm{du}$ cheptel de la préfecture du lac.

La streptothricose se manifeste sous une forme enzootique et une allure bénigne.

Quelques rares cas de pasteurellose ont été signalés.

Les améliorations sanitaires doivent être complétées par :

- la destruction des fauves, principalement des hyènes et des chacals, qui sont responsables de la perte de 4 à 5 p. 100 de jeunes animaux et de bovins hors d'âge;

- la lutte contre les feux de brousse qui détruisent totalement le tapis herbacé jus- qu'à la saison des pluies suivante, d'où l'insuffisance de pâturages, des maladies par carences etc.

\section{CONCLUSION}

La race Kouri semble avoir été dans le passé l'une des meilleures du Tchad. Aujourd'hui cette race, en voie de disparition tant au Niger qu'au Tchad, a régressé en qualité zootechnique et en nombre; on estime son effectif à une cinquantaine de milliers d'individus.

Parmi les économistes, les uns prétendent que tout doit être mis en cuvre pour préserver et améliorer la race; d'autres affirment que sa faible importance économique, par rapport à l'ensemble du cheptel tchadien, ne nécessite pas actuellement sa relance.

$\mathrm{Du}$ point de vue scientifique, les zootechniciens estjment qu'il serait très souhaitable de favoriser la persistance d'un noyau d'élevage Kouri à l'état pur. En effet, les qualités intéressantes de cette race, si bien adaptée à son milieu, sont susceptibles, par la sélection, d'améliorer les performances, qui n'ont encore été que partiellement exploitées. Enfin, l'obtention de métis de $1^{\text {re }}$ génération, dont les performances semblent prometteuses, nécessite le maintien de Kouris purs sélectionnés.

\section{SUMMARY}

\section{The "Kouri ": A cattle breed from lake Chad}

I - A general survey of the breed, with reference to zootechnical and biochemical studies, its origins and ecology

The authors provide an exhaustive study of the breed of the Kouri cattle (Bos taurus), a breed of horned cattle from lake Chad.

That study is meant as an introduction to the whole field of research work tending to clarify the different features, both zootechnical and biochemical, of that particular breed which has been known for a long time and is perfectly adapted to the aquatic environment in lake Chad.

The object in view is to save from extinction a link, most likely an essentiel one, in the chain of the phylogeny of the cattle breeds, and to make immediate use of the most valuable assets of that breed, viz. (namely) its high yield of milk and choice meat, together with the specific qualities of the cross-breeds of 1 st or 2 nd generation.

Following the ecology of the breed, and the location of its natural habitat, there comes a description of the standard type and its specific qualities, considered from the economic angle.

Then the authors deal very thoroughly with the methods of breeding and the pathology of the breed.

At the moment there are many reasons in favour of the continuation and selection of the pure bred "Kouri". 


\section{RESUMEN}

El a Kouri n : raza bovina del lago Chad

I - Estudio general zootécnica y bioquimica, origen

y ecologia de la raza

Detalladamente se describe la raza « Kouri » (Bos taurus) bovinos del lago Tchad. Este estudio es una introducción del conjunto de trabajos teniendo por objeto el mejor conocimiento de los varios aspectos zootécnicos y bioquimicos de dicha raza conocida desde hace ya mucho tiempo y estrechamente ligada con el ambiente acuático del lago Chad.

El propósito perseguido es el amparo de un eslabón, sin duda esencial, en la filogénesis de las razas bovinas y el aprovechar rápido de las propiedades muy interesantes de la raza: producción lechera, producción de carne de primera clase y cualidades de los mestizos de primera o de segunda generación.

Después del estudio de la ecologia y de la repartición geográfica de la raza, se describe el tipo característico y sus cualidades desde el punto de vista económico. Se indican con detalle el modo de ganaderia y la patologia.

Actualmente, numerosas razones hablan en pro para el mantenimiento y la selección de los bovinos de pura raza « Kouri».

\section{BIBLIOGRAPHIE}

1. BOUCHARDEAU (A.), LEFEVRE (R.), Monographie du lac Tchad. Tome I. O.R.S.T.O.M., 1957 (Commission Scientifique du Logone et du Tchad).

2. CURSON (J.), THORNTON (W.), A contribution to the study of african native cattle, Onderstepoort, J. vet. Sci., 1936, 7 (2): 613.

3. DOUTRESSOULLE (G.), L'élevage en Afrique Occidentale Française, Paris, Larose, 1947.

4. GASTON (A.), Etude agrostologique du Kanem. Préfecture du Kanem au sud du 16e parallèle et préfecture du lac. Maisons-Alfort, I.E.M.V.T., 1967,147 p., 1 carte en coul. 1/500.000e (Etude agrostologique n* 19).

5. GENTIL (P.), Les treize préfectures de la République du Tchad, 1962.

6. GRABER (M.), Helminthes et helminthiases. Bilan d'activité. Région de recherches vétérinalres et zootechniques d'Afrique centrale, rapport annuel 1967, tome 3, Farcha, Fort-Lamy, I E.M.V.T.

7. JOSHI (R.), McLAUGHLIN (A.), PHILLIPS (W.), Les bovins d'Afrique. Types et races. Rome, F.A.O., 1957 (Etudes agricoles no 37).

8. KONE (K.), Le bouf du lac Tchad de la région de N'Guigmi, Bull. Serv. Elev. Ind. Anm. A.O.F., 1948, 1 (2): 47-65.

9. LACHAUX (P.), LEVIF (J.), TROUETTE (M.), Etude sur les abattages de bovins à l'abattoir frigorifique de Farcha, Fort-Lamy, statistique 1967.

10. LAPLANCHE (S.), L'élevage transhumant en zone sahélienne, Maisons-Alfort, «Au Manuscrit », 1969. Thèse. Doct. vét. Paris, 1969, $\pi^{\text {' } 105 .}$

11. MALBRANT (R.), RECEVEUR (P.), SABIN (R.), Le bouf du lac Tchad, Rev. Elev. Méd. v'ét. Pays trop., 1947, 1 (1) . 37-42; (2) : 109-29.

12. MARTIN, Etude sur les abattages de bovins à l'abattoir frigorifique de Farcha à Fort-Lamy. Statistiques 1968.

13. MOREL (P. C.), GRABER (M.), Les tiques des animaux domestiques du Tchad, Rev. Elev. Méd. vét. Pays trop., 1961, 14 (2): 199-203.

14. PIAS (J.), GUICHARD (E.), Etude pédologique des rives du lac Tchad de Djimtilo à Bol et du sillon du Bahr el Ghazal de Massakory à Moussoro, O.R.S.T.O.M.. Centre de Recherches tchadiennes, 1960.

15. Rapport du Service de l'Elevage du Tchad, 1964.

16. SARNIGUET (J.), MARTY (J. P.), ARNAUD (R.), Exploitation du cheptel bovin au Tchad. Tome I, Paris, Secrétariat d'Etat aux Affaires étrangères chargé de la Coopération, 1967.

17. STEWART (J. L.), The cattle of the gold Coast, Emp. J. exp. Agric, 1938.

18. TOUCHEBEUF de LUSSIGNY (P.) et Collab., Monographie hydrologique du lac Tchad, FortLamy, O.R.S.T.O.M., 1969. 